\title{
Decay of hadronic resonances in the magnetic field
}

\section{Peter Filip ${ }^{* \dagger}$}

Institute of Physics, Slovak Academy of Sciences, Dúbravská cesta 9, Bratislava 845 11, Slovakia E-mail: Peter.Filip@savba.sk

\begin{abstract}
We investigate, to what extent the external magnetic field can modify decay channels of mesons and hadronic resonaces. Magnetic fields $10^{14}-10^{15} \mathrm{~T}$, created in ultra-relativistic collisions of heavy nuclei are considered, and their influence on $\rho^{0}, \Lambda^{*}(1520), K^{*}, D^{*}$ and $\Delta^{0}$ resonant states is studied. We find that $\Lambda^{*}, \Xi^{0 *}$ and $\Delta^{0}(1232)$ decays are affected by field $B \approx 5 \cdot 10^{14} \mathrm{~T}$ and $K^{*} \rightarrow K^{ \pm} \pi^{\mp}$ decays are influenced in field $B \approx 10^{15}$ T. Decay channel $D^{ \pm *} \rightarrow D^{0}+\pi^{ \pm}$becomes suppressed at static field $3 \cdot 10^{13} \mathrm{~T}$, but relatively long lifetime of $D^{ \pm *}$ meson prevents this effect to be relevant in heavy ion collisions. In the case of $\rho^{0}$ meson we suggest that enhanced production of photons and dileptons from $\rho^{0}$ decays may happen in heavy ion collisions due to kinematical suppression of strong $\rho^{0} \rightarrow \pi^{+} \pi^{-}$decay.
\end{abstract}

XXII International Baldin Seminar on High Energy Physics Problems

15-20 September, 2014

JINR, Dubna, Russia

\footnotetext{
* Speaker.

${ }^{\dagger}$ With special thanks to organizers for their support.
} 


\section{Introduction}

Magnetic field created in the interaction region of heavy ion collisions [1] at RHIC and LHC may reach values $10^{14}-10^{15} \mathrm{~T}$ for a short time. Presence of so large external field in the interaction volume of colliding nuclei is of particular interest, since the behavior of the expanding partonic matter [2] and parameters of phase transition into the hadronic final state [3] can be influenced. In ultra-central collisions of nuclei, with very small $(b \approx 0)$ impact parameters, magnetic fields in the interaction region may become reduced to smaller values, which gives us the possibility to test experimentally the sensitivity of dense QCD matter to extremal magnetic fields.

It has been suggested [4], that lifetime of the magnetic field created in the relativistic collision of heavy nuclei can be substantialy prolonged (from $\tau=0.1 \mathrm{fm} / \mathrm{c}$ up to $3-5 \mathrm{fm} / \mathrm{c}$ ) due to the presence of charged partonic plasma, which is able to "freeze" the magnetic flux in the interaction region. Therefore, decays of $\rho(770)$ mesons and $\Delta(1232)$ resonances (with lifetimes $\tau_{\rho}=1.3 \mathrm{fm} / \mathrm{c}$ and $\left.\tau_{\Delta}=1.7 \mathrm{fm} / \mathrm{c}\right)$, as well as $K^{*}$ mesons $\left(\tau_{K^{*}}=\approx 4 \mathrm{fm} / \mathrm{c}\right)$ and possibly also $\Lambda^{*}(1520)$ baryons with $\tau_{\Lambda^{*}} \approx 13 \mathrm{fm} / \mathrm{c}$, may thus be influenced due to their relatively short lifetimes, if coexistence of partonic and hadronic phases in heavy ion collisions is assumed.

\section{Energy of Charged Particles in Magnetic Field}

Modification of partial decay width of $\rho^{0} \rightarrow \pi^{+} \pi^{-}$channel (due to Landau level energy increase for charged decay products) has been considered in work [5], where possibility of $\rho^{ \pm}$meson condensation in static fields $B \approx 10^{16} \mathrm{~T}$ has been suggested.

Let us describe here in a more detail the mechanism influencing the probability of a resonance decay to charged daughter particles in the magnetic field: Energy of charged particles in the magnetic field is quantized [6] and for the lowest $(n=0)$ Landau level of particle with spin and charge $(Q=e)$ we have [5]

$$
E[B]=\sqrt{m^{2}+p_{z}^{2}+e B\left(1-2 s_{z}\right)} .
$$

For $p_{z} \ll m^{2}$ and $e B \ll m^{2}$ one obtains

$$
E[B] \approx m+\left(p_{z}^{2}+e B\right) / 2 m-e B s_{z} / m
$$

From Eq.(2.2) we observe that energy of spinless $\left(s_{z}=0\right)$ charged particles $\left(\pi^{ \pm}, K^{ \pm}\right)$increases with the magnetic field by amount $\Delta E_{L}=e B / 2 m$. Due to Landau level $\Delta E_{L}$ shift the effective mass of decay products (in the magnetic field) may reach values larger than rest mass $M_{R^{*}}$ of decaying resonace. The resonace $R^{*}$ then cannot decay into that particular decay channel (e.g. $\rho^{0}(770) \rightarrow \pi^{+} \pi^{-}$, as shown in Fig. 1) and its decay width may become considerably reduced.

\section{Decay of $\rho^{0}(770)$ Meson in the Magnetic Field}

In the case of $\rho^{0}(770)$ meson, closing of $\pi^{+} \pi^{-}$decay in the magnetic field may have quite interesting consequences: Since the second possible strong decay channel $\rho^{0}(770) \rightarrow \pi^{0} \pi^{0}$ is 
forbidden by the isospin and $\mathrm{C}$ parity conservation, the probability of $\rho^{0}$ decay via remaining channels $\left(\rho^{0} \rightarrow \pi^{0} \gamma, \rho^{0} \rightarrow \eta \gamma, \rho^{0} \rightarrow \pi^{0} \pi^{0} \gamma\right.$, and $\rho^{0} \rightarrow e^{+} e^{-}$or $\left.\rho^{0} \rightarrow \mu^{+} \mu^{-}\right)$is enhanced by factor $\approx 10^{3}$ compared to the usual branching ratio values. This may lead to considerable excess of photons and dileptons (in heavy ion collisions) above the expected yields. Indeed, if only a small fraction (e.g. 5\%) of $\rho^{0}$ mesons created in heavy ion collisions cannot decay into channels containing $\pi^{+} \pi^{-}$pair, dilepton yield from $\rho^{0} \rightarrow l^{+} l^{-}$decays is boosted by factor $5 \mathrm{x}$. Critical field for $\rho^{0} \rightarrow \pi^{+} \pi^{-}$decay can be estimated from Eq.(2.1) as $B_{c} \approx 2.2 \cdot 10^{15} \mathrm{~T}$, if one assumes that internal structure (mass and magnetic moment) of $\rho^{0}$ meson remain unchanged in the field.

Since only one charged pion is produced in $\rho^{ \pm} \rightarrow \pi^{ \pm} \pi^{0}$ decays, the critical field for $\rho^{ \pm}$ decays is larger: $B_{c}=3.8 \cdot 10^{15} \mathrm{~T}$ (see Fig.1). The energy of $\rho_{m_{z}=1}^{+}$meson state decreases (as shown in Fig.1) due to its magnetic moment interaction $E=-\mu \cdot B$ with external field $B$, which is taken into account by term $-2 e B s_{z}$ in Eq.(2.1). The last term in Eq.(2.2) $E_{i n t}=-s_{z} B e / m$ is equal to $-\mu \cdot B$, if one assumes $\mu=\hbar Q / 2 m$ for charged $(Q=e)$ particle with spin $s_{z}=\hbar / 2$.

In case $s_{z}=0$, the energy of charged $\rho^{+}$is still increasing in the magnetic field due to Landau level $\Delta E_{L}$ shift, and for $m_{z}=-1$ the energy rises also due to $E=+B \cdot \mu_{\rho^{+}}$interaction term. Lines for $\rho_{m_{z}=0}^{+}$and $\rho_{m_{z}=-1}^{+}$are not shown in Fig.1. We also do not discuss here the phenomenon of $\rho^{ \pm}$ meson condensation predicted in [5] to occur at $B_{c} \approx 10^{16} \mathrm{~T}$.

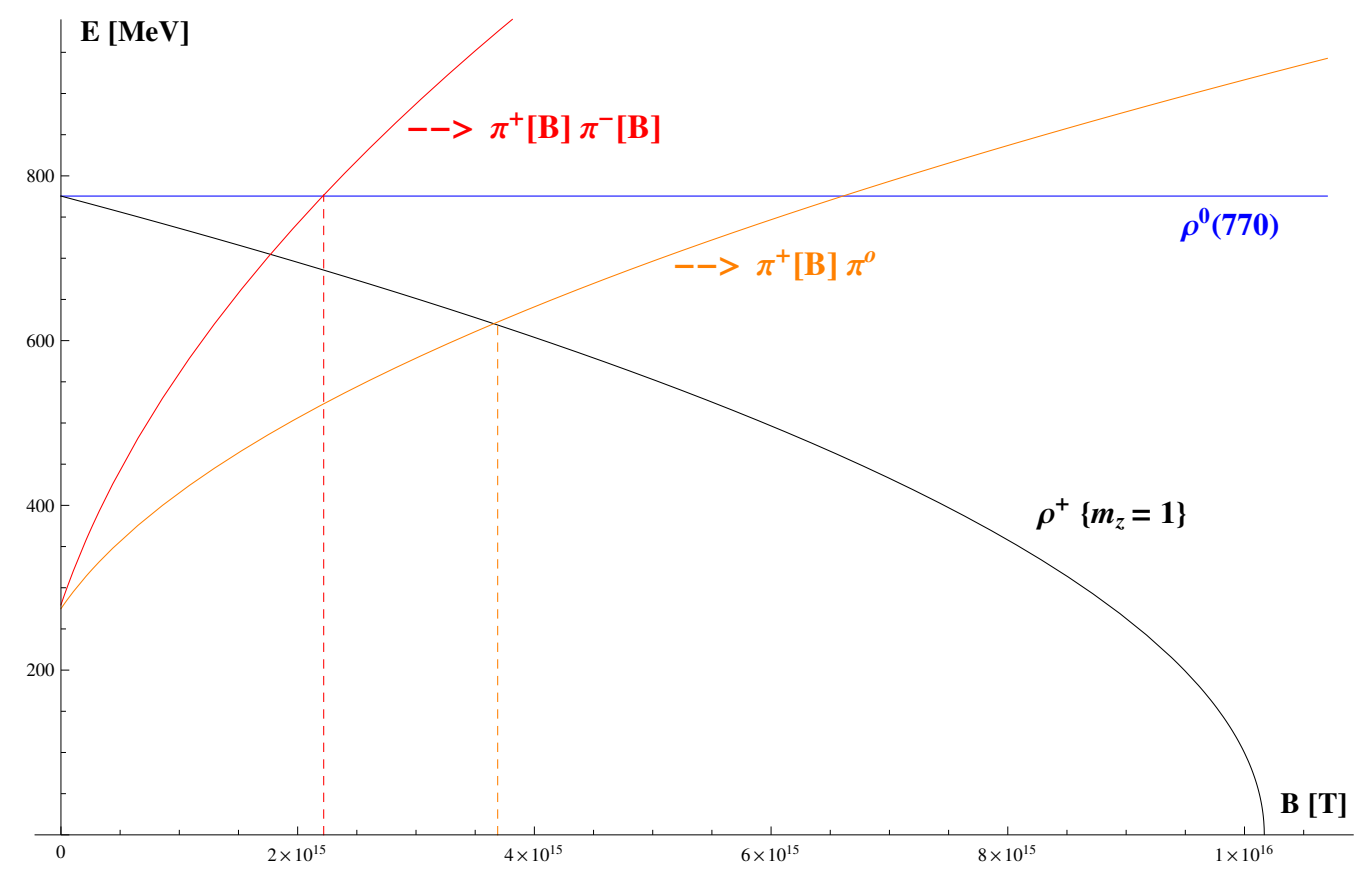

Figure 1: Energies of $\rho^{0}$ and $\rho_{m_{z}=1}^{+}$mesons and of their $\pi^{+} \pi^{-}$and $\pi^{+} \pi^{0}$ decay products in static magnetic field, calculated assuming $\mu_{\rho^{+}}=2.42 \mu_{N}$, according to Ref. [5].

\section{Decay of Neutral $K^{*}$ Mesons in the Magnetic Field}

Neutral hadrons with non-zero spin are composite particles, and they may possess magnetic moments (e.g. $\mu_{\Lambda^{0}}=-0.61 \mu_{N}$ ), which can interact with the magnetic field. In such cases, expres- 
sion (2.1) needs to be reformulated, for taking into account the possibility of $Q=0$ and $\mu \neq 0$. One can tentatively assume $\Delta E_{L}=0$ and simply write $E[B]=\sqrt{m^{2}+p_{z}^{2}+e B\left(-2 s_{z}\right)}$ for a neutral particle with the magnetic moment $\mu=\hbar e / 2 m$. However, this is not satisfactory, since real magnetic moment of neutral (and also charged) hadrons deviates from Dirac value $\mu=\hbar e / 2 m$ due to their compositness. (For proton, $\mu_{\tilde{p}}=e \hbar / 2 m_{p}=\mu_{N}=3.15 \cdot 10^{-8} \mathrm{eV} / \mathrm{T}$ could be naively expected, but measured value is $\mu_{p}=2.79 \mu_{N}$.) For this reason, we rewrite Eq.(2.1) in the following form

$$
E[B]=\sqrt{m^{2}+p_{z}^{2}+2\left|q_{e}\right| \mu_{N} m_{p} B-2 J_{z} \mu_{N} \tilde{\mu}_{\left[\mu_{N}\right]} m B}
$$

where $q_{e}$ is charge of hadron in units $e=1.602 \cdot 10^{-19} \mathrm{C}$ (for $\Delta^{++}(1232) q_{e}=2$, and for proton $\left.q_{e}=1\right), \tilde{\mu}_{\left[\mu_{N}\right]}$ is magnetic moment of hadron in $\mu_{N}$ units (for proton $\tilde{\mu}=2.79$ ), $J_{z}=-1,0,1$ is the spin "projection" value, and $m_{p}=938.27 \mathrm{MeV}$ (proton mass). This equation allows one to calculate the energy of neutral or charged particle in the magnetic field using the real magnetic moment $\tilde{\mu}$ of the particle. Indeed, for $p_{z} \ll m^{2}$ and $e B \ll m^{2}$ one obtains the expected behavior

$$
E[B]=m+p_{z}^{2} / 2 m+\left|q_{e}\right| e B / 2 m-J_{z} \mu_{N} \tilde{\mu}_{\left[\mu_{N}\right]} B
$$

since the energy of magnetic moment $\tilde{\mu}$ in external field is $E=-\tilde{\mu} \cdot B\left(J_{z}\right.$ values $+1,-1$ are used here also for hadrons with $s_{z}=1 / 2$ ). Landau energy shift $\Delta E_{L}$ in Eq.(4.2) is the same as in Eq.(2.2).

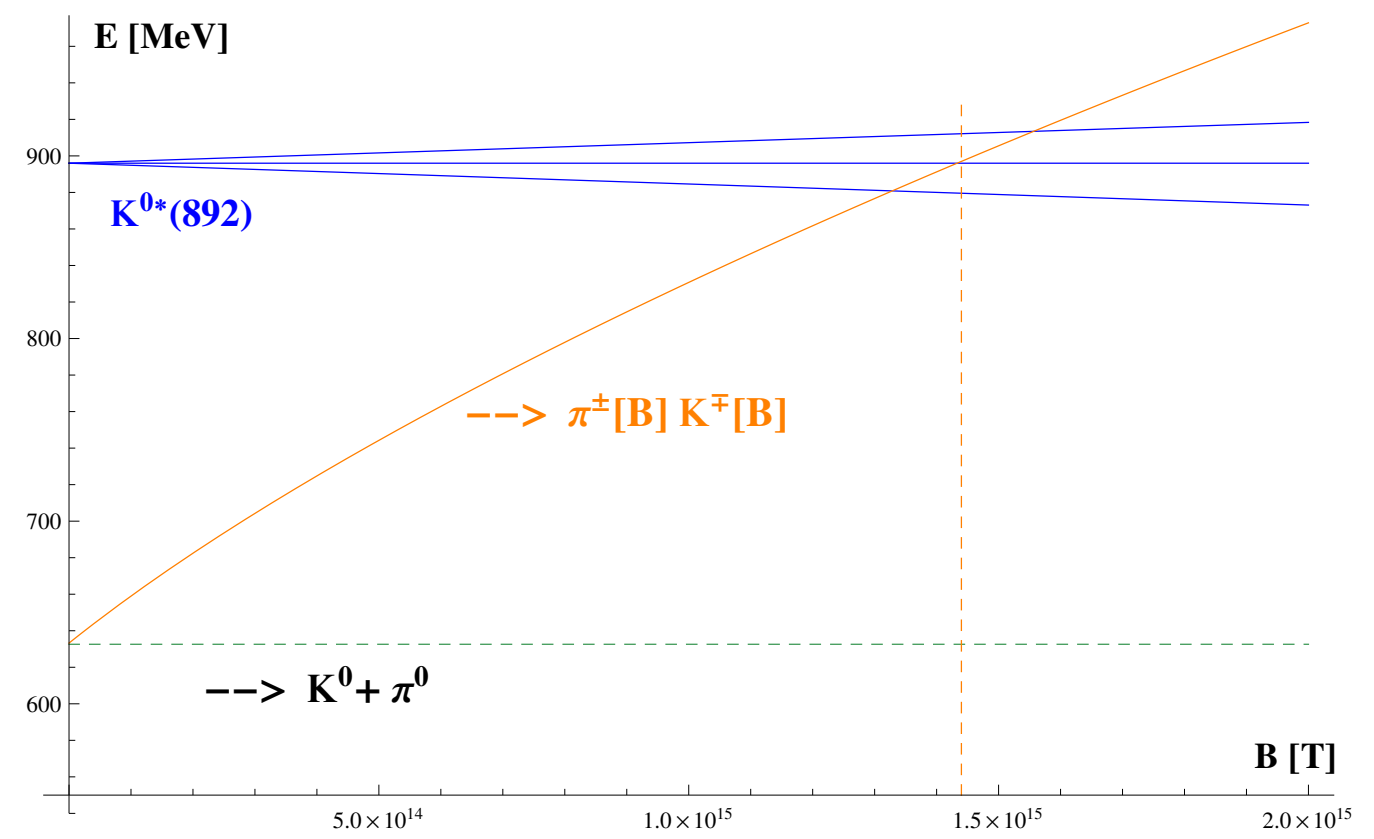

Figure 2: Energy of $K^{0 *}(892)$ meson $\left(m_{z}= \pm 1,0\right)$ substates and of its decay products $K^{ \pm}[B]+\pi^{\mp}[B]$ in the magnetic field according to Eq.(4.1), assuming $\mu_{K^{0 *}}=\left(\mu_{d}+\mu_{\bar{s}}\right)=-0.36 \mu_{N}$.

The energy of $\left(K^{ \pm} \pi^{ \pm}\right)$particle pair may grow in the magnetic field (due to $n=0$ Landau level increase) above the $K^{0 *}$ mass, and decay $K^{0 *} \rightarrow K^{ \pm} \pi^{ \pm}$becomes kinematically forbidden in field $B \approx 1.4 \cdot 10^{15} \mathrm{~T}$ (see Fig.2). At the same time, decay into neutral daughter products $K^{0}+\pi^{0}$ is unaffected by the magnetic field (in our approximation). 
When total yield of $K^{0 *}(892)$ mesons (e.g. in $\mathrm{Pb}+\mathrm{Pb}$ collisions at LHC [7]) is determined from $\bar{K}^{0 *}, K^{0 *} \rightarrow K^{ \pm} \pi^{\mp}$ decays assuming standard isospin-conserving branching ratio (66\%), the influence of the large magnetic field [1] may systematically bias the extracted yield values, because reduced phase space for $K^{0 *} \rightarrow K^{ \pm} \pi^{\mp}$ decays leads to smaller branching ratios than expected.

Decay of charged $K^{ \pm *}$ mesons in the static magnetic field is influenced differently, because magnetic moment $\mu_{K^{ \pm *}}= \pm\left(\mu_{u}+\mu_{\bar{s}}\right)= \pm(1.85+0.61)= \pm 2.46 \mu_{N}$ is $5 \mathrm{x}$ larger compared to $\mu_{K^{0 *}}=\left(\mu_{d}+\mu_{\bar{s}}\right)=-0.36 \mu_{N}$. Triplet energy splitting for $K^{ \pm *}$ in the magnetic field is large and critical field $B_{c}$ which would close $K^{ \pm *} \rightarrow K^{0}+\pi^{ \pm}$decay channel completely does not exist.

\section{Decay of $\Lambda^{*}(1520)$ Baryon in the Magnetic Field}

Magnetic moment of $\Lambda^{*}(1520)$ resonance $\left(J=3 / 2^{-}\right)$state is expected to be non-zero [8], and therefore, in external magnetic field, the energy of $\Lambda^{*}(1520)$ baryon splits into four lines, as shown in Fig.3 (we assume here $\mu_{\Lambda^{*}} \approx-0.2 \mu_{N}$ ). For two decay channels $\Lambda^{*}(1520) \rightarrow p+K^{-}(22.5 \%)$ and $\Lambda^{*}(1520) \rightarrow \Lambda^{0} \pi^{+} \pi^{-}$(10\%) we have calculated the Landau level energies using Eq.(4.1). Decay to $\Lambda^{0} \pi^{+} \pi^{-}$becomes kinematically forbidden in field $B>3.5 \cdot 10^{14} \mathrm{~T}$ (see Fig.3). There are two lines corresponding to the energy of $\Lambda^{0} \pi^{+} \pi^{-}$decay products in Fig.3, because magnetic moment $\left(\mu_{\Lambda^{0}}=-0.61 \mu_{N}\right)$ interaction of $\Lambda^{0}$ baryon with the magnetic field splits the energy of its two $s_{z}=+1 / 2$ and $s_{z}=-1 / 2$ substates into a doublet.

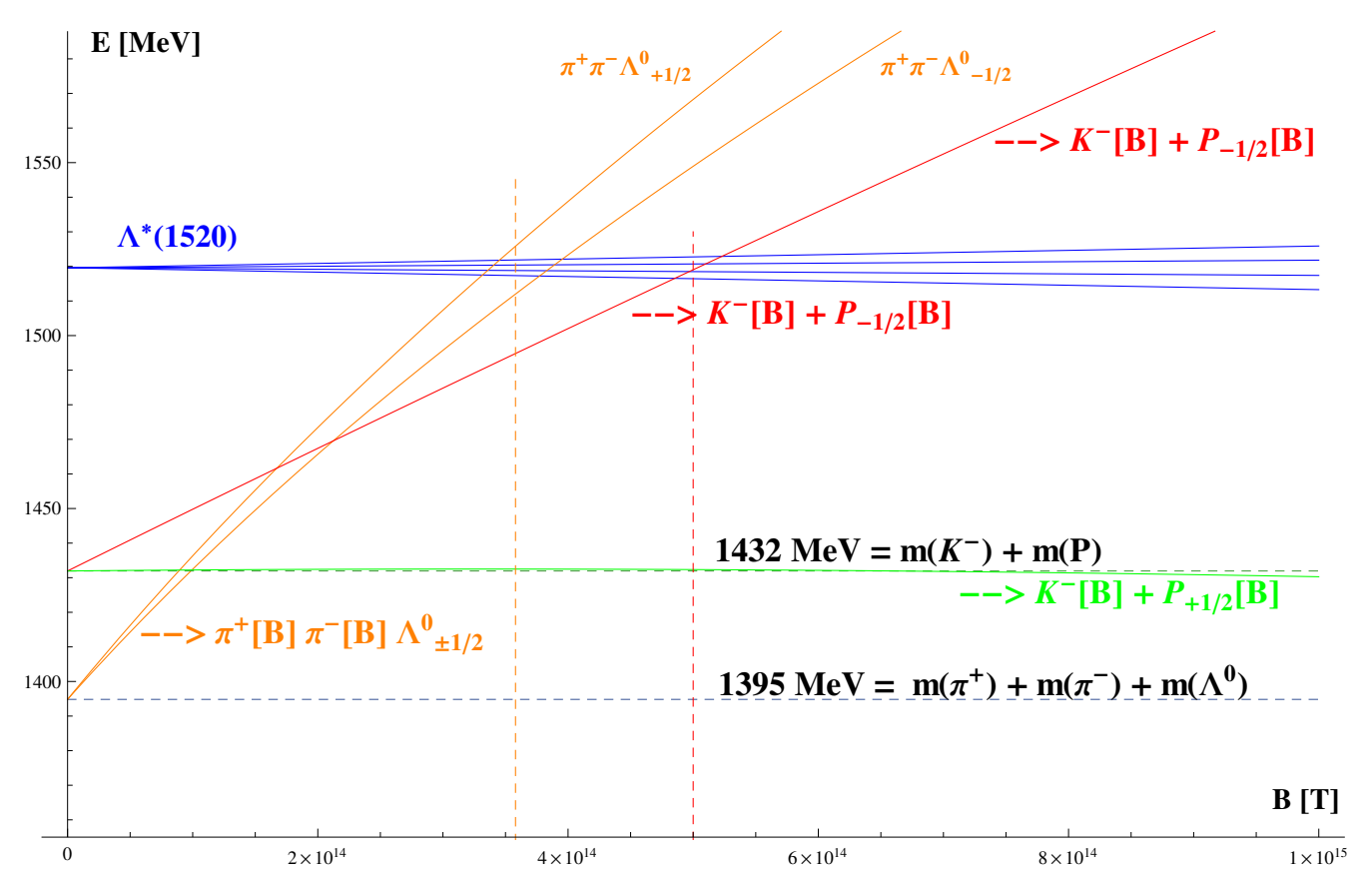

Figure 3: Energy of $\Lambda_{3 / 2}^{*}(1520)$ baryon $\left(s_{z}= \pm 1 / 2, \pm 3 / 2\right)$ substates (using $\mu_{\Lambda^{*}}=-0.2 \mu_{N}$ [8]) and of its decay products for channels $\Lambda^{*} \rightarrow K^{-} p^{+}$and $\Lambda^{*} \rightarrow \Lambda^{0} \pi^{+} \pi^{-}$in the magnetic field.

According to isospin conservation, $\Lambda^{*}(1520) \rightarrow N+K$ decay proceeds in vacuum via charged $\left(p+K^{-}\right)$and neutral $\left(n+\bar{K}^{0}\right)$ channels with equal probabilities $(22.5 \%$ and $22.5 \%)$. Energy of 
decay products in the neutral channel is affected only by the interaction of neutron magnetic moment $\mu_{n}=-1.9 \mu_{N}$ with external field. For $B \leq 10^{15} \mathrm{~T}$ we have energy shift $\Delta E=|\mu \cdot B| \leq 60 \mathrm{MeV}$, which is not enough to close kinematically this decay channel (mass difference between $\Lambda^{*}(1520)$ and neutral decay products $\left(M_{K}+M_{n}=1437 \mathrm{MeV}\right)$ is larger: $\left.\Delta M_{K n}^{\Lambda^{*}}=82 \mathrm{MeV}\right)$.

However, energy of proton with its spin down $\left(s_{z}=-1 / 2\right)$ is increasing due to term $-\mu_{p} \cdot B$ in Eq.(4.2) and also due to its Landau shift $\Delta E_{L}=e B / 2 m_{p}$. Moreover, Landau level $\Delta E_{L}=e B / 2 m_{K}$ of charged Kaon grows almost $2 \times$ faster compared to proton $\Delta E_{L}$, due to significantly smaller Kaon mass. Total energy of $K^{-}+{ }^{\downarrow} p_{-1 / 2}$ decay products thus becomes larger than $\Lambda^{*}(1520)$ mass at $B \approx 5 \cdot 10^{14} \mathrm{~T}$ (see rising red line starting at $1432 \mathrm{MeV}$ in Figure 3).

For decay channel $\Lambda^{*} \rightarrow K^{-}+{ }^{\uparrow} p_{1 / 2}$, the energy of Proton with spin up $\left(s_{z}=1 / 2\right)$ is decreasing (magnetic moment of proton is positive), but this decrease is compensated by the Kaon and proton Landau level energy increase $\left(\Delta E_{L}=e B / 2 m\right)$, which results in almost horizontal (green) line denoted as $\rightarrow K^{-}[B]+P_{+1 / 2}[B]$ in Fig.3.

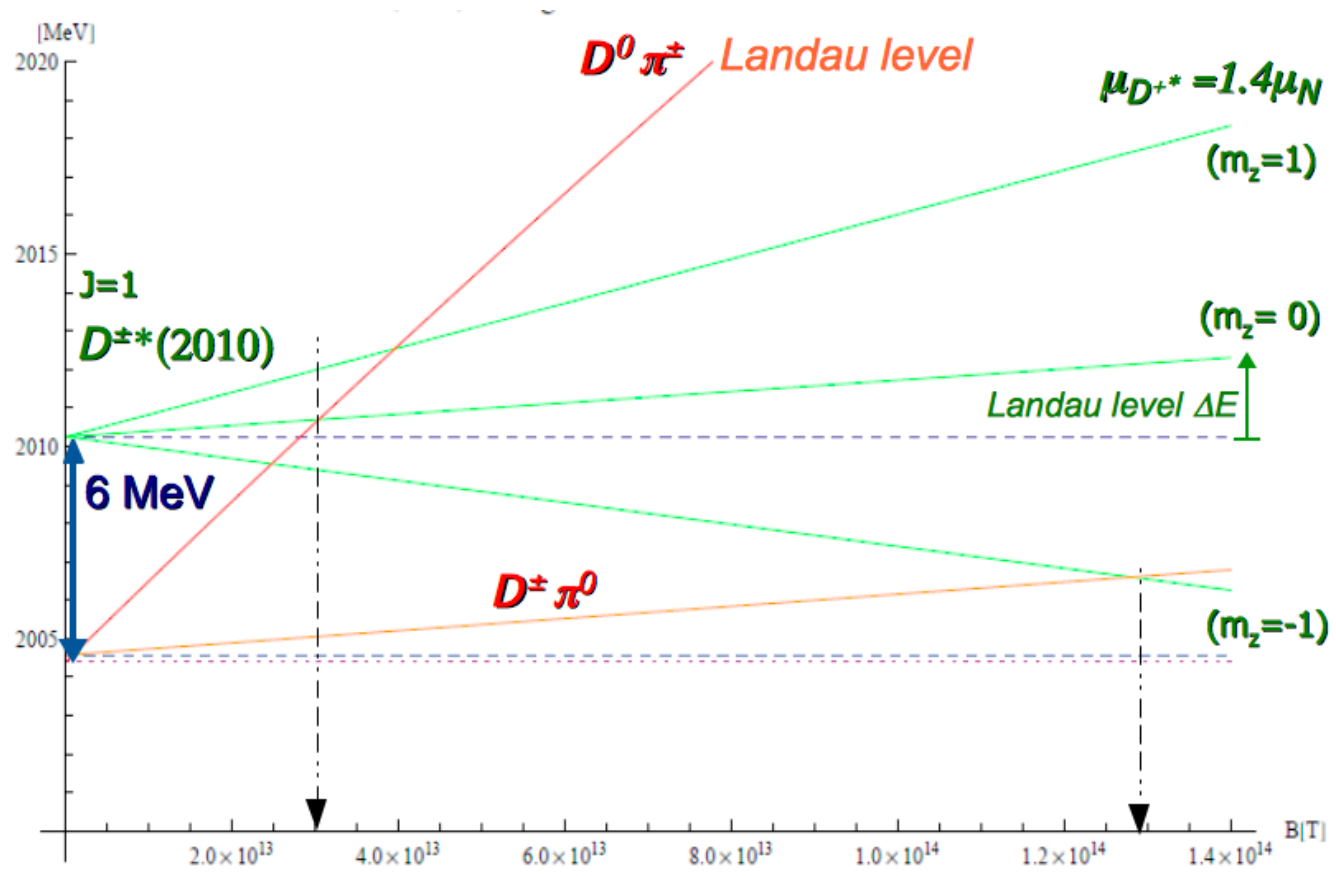

Figure 4: Energy of $D^{ \pm *}(2010)$ meson $s_{z}= \pm 1,0$ substates (assuming $\left.\mu_{D^{ \pm *}}= \pm\left(\mu_{c}+\mu_{\bar{d}}\right)= \pm 1.37 \mu_{N}\right)$ and of its decay products $D^{ \pm *} \rightarrow D^{ \pm} \pi^{0}$ and $D^{ \pm *} \rightarrow D^{0} \pi^{ \pm}$in static magnetic field $B \approx 10^{14} \mathrm{~T}$.

Therefore, $\Lambda^{*} \rightarrow \Lambda^{0} \pi^{+} \pi^{-}$decay becomes kinematically closed in field $B>3.5 \cdot 10^{14} \mathrm{~T}$, and $50 \%$ of $\Lambda^{*} \rightarrow K^{-} p^{+}$decays become suppressed in $\approx 5 \cdot 10^{14} \mathrm{~T}$ field. However, lifetime $\tau \approx 13 \mathrm{fm} / \mathrm{c}$ of $\Lambda^{*}(1520)$ is relatively long compared to the expected duration of the magnetic field in heavy ion collisions. If freezing of the magnetic flux [4] in deconfined (QGP) medium [9] is assumed, reconstructed $\Lambda^{*}(1520)$ yields in ultra-relativistic collisions of heavy nuclei might be influenced by extremal magnetic fields [1] created in such interactions, provided that coexistence of partonic and hadronic phases is considered. Experimental data [10] on $\Lambda^{*}(1520)$ production in Au+Au collisions at RHIC allow one to speculate, that decrease of $\Lambda^{*} / \Lambda$ ratio observed in peripheral $\mathrm{Au}+\mathrm{Au}$ collisions and disappearance of $\Lambda^{*}$ signal in non-central collisions [10] might be followed by the 
increase of $\Lambda^{*} / \Lambda$ ratio in central collisions, where $\Lambda^{*}$ signal is again observed. It is rather tempting to suggest, that magnetic field influence may by involved, because in non-central collisions, the created magnetic field and its duration are the largest. Improved statistics for the measurement of $\Lambda^{*}$ production in $\mathrm{Au}+\mathrm{Au}$ collisions [11] at RHIC would clarify the observed $\Lambda^{*} / \Lambda$ behavior [10].

\section{Decay of $D^{ \pm *}(2010)$ Mesons in the Magnetic Field}

Lifetime of charged $D^{ \pm *}(2010)$ mesons $\tau_{D^{*}} \approx 2000 \mathrm{fm} / \mathrm{c}$ is much larger than the expected duration of the magnetic field existence in have ion collisions. Nevetherless, in order to satisfy our curiosity and also for the completeness, we show the expected behavior of $D^{ \pm *}$ mesons subject to magnetic field in Fig. 4. The critical field $B_{c} \approx 3 \cdot 10^{13} \mathrm{~T}$ in which $D^{ \pm *} \rightarrow D^{0}+\pi^{ \pm}$is kinematically closed is two orders of magnitude smaller than the critical field for $\rho^{0}$ and $K^{0 *}$ mesons. This is a simple consequence of the small mass difference $\Delta M=6 \mathrm{MeV}$ between $D^{ \pm *}$ meson and its decay product $(D+\pi)$. Channel $D^{ \pm *} \rightarrow D^{ \pm}+\pi^{0}$ does not become closed in the magnetic field, because Landau energy increase $\Delta E_{L}$ for massive $D^{ \pm}(1870)$ mesons is much smaller compared to $\pi^{ \pm}$case.

\section{Decay of $\Xi^{0 *}(1535)$ in the Magnetic Field}

Behavior of $\Xi^{0 *}(1535)$ baryonic state in the magnetic field might be relevant for heavy ion collisions, although the reduced yield of $\Xi^{0 *}$ mesons in Au+Au interactions at RHIC seems to be understood [12]. Critical field for full suppression of $\Xi^{0 *} \rightarrow \Xi^{-}+\pi^{+}$decay is $B_{c}=4 \cdot 10^{14} \mathrm{~T}$.

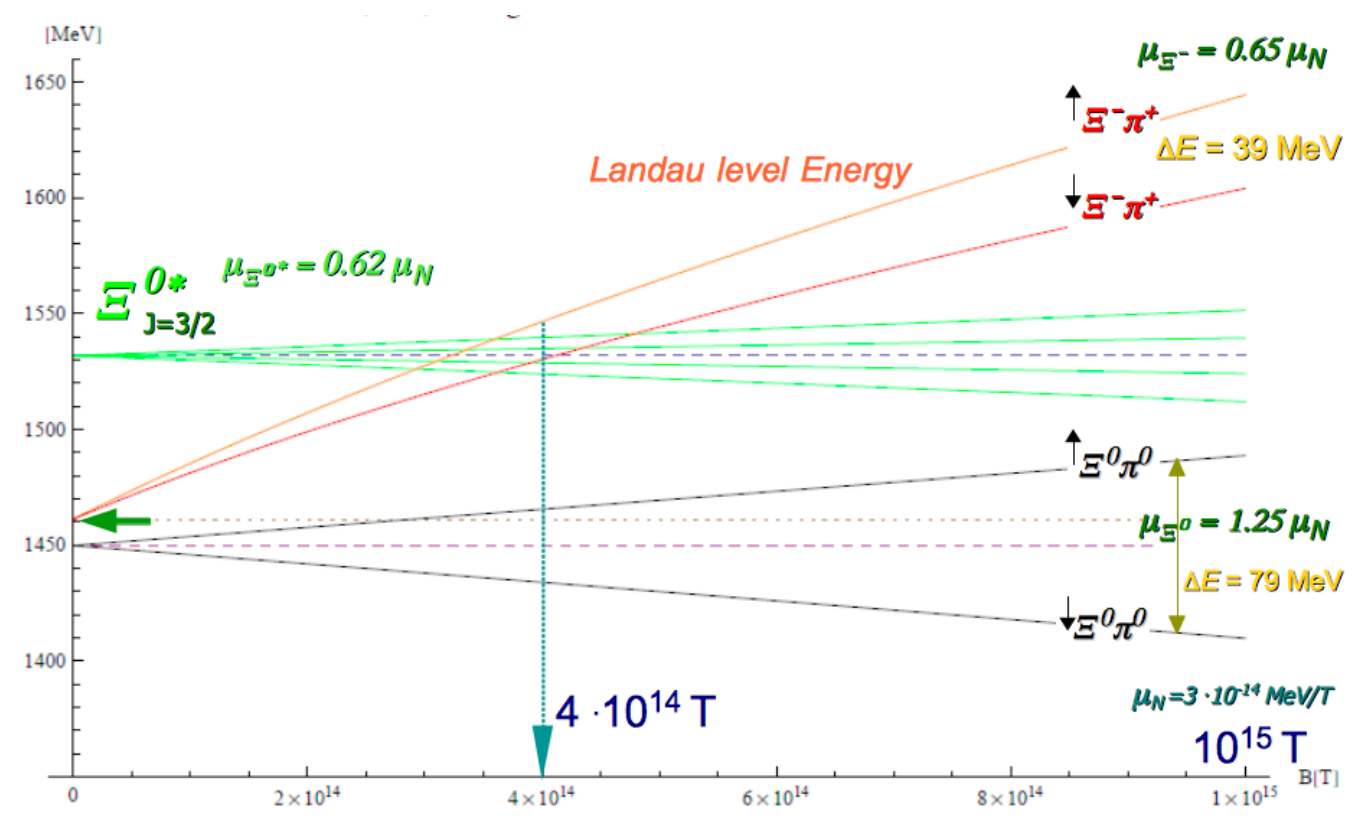

Figure 5: Energy of $\Xi^{0 *}(1532)$ baryon $\left(s_{z}= \pm 1 / 2, \pm 3 / 2\right)$ and of its decay products $\Xi^{-} \pi^{+}$and $\Xi^{0} \pi^{0}$ in static magnetic field (using $\mu_{\Xi 0 *}=2 \mu_{s}+\mu_{u}=0.62 \mu_{N}$ and orientations $s_{z}= \pm 1 / 2$ for $\Xi^{-}$and $\Xi^{0}$ ). 
Although critical magnetic field for $\Xi^{0 *} \rightarrow \Xi^{-}+\pi^{+}$channel is comparable to the critical field for $\Lambda^{*}(1520) \rightarrow p+K^{-}$decay, it is neccessary to point out, that lifetime $\tau_{\Xi^{0 *}} \approx 21 \mathrm{fm} / \mathrm{c}$ of $\Xi^{0 *}$ baryon is almost two times larger than lifetime of $\Lambda^{*}$, and many times larger than the expected duration of magnetic fields created in heavy ion collisions. However, $\Lambda^{*} \rightarrow K^{-}+p$ decays are suppressed only by $50 \%$, while $\Xi^{0 *} \rightarrow \Xi^{-}+\pi^{+}$decays are $100 \%$ suppressed in field $B \approx 5 \cdot 10^{14} \mathrm{~T}$, and therefore, the influence of the static magnetic field - if present - might be comparable.

For the magnetic moment of $\Xi^{0 *}$ we use value $\mu_{\Xi^{0 *}}=\left(\mu_{u}+2 \mu_{s}\right)=1.85-1.22=0.62 \mu_{N}$ assuming simple additive rule for $J=3 / 2^{+}$baryonic state with parallel spins of constituent quarks. For magnetic moments of $\Xi^{0}$ and $\Xi^{-}$, which are responsible for the splitting of Landau energy of decay products (as shown in Fig. 5), we use the measured values $1.25 \mu_{N}$ and $0.65 \mu_{N}$.

\section{Decay of $\Delta^{0}(1232)$ Resonance in the Magnetic Field}

Resonant state $\Delta^{0}$ with parallel spins of three constituent quarks (uud) decays strongly to $p+\pi^{-}$and $n+\pi^{0}$ decay products with probabilities $33 \%$ and $66 \%$ determined by the isospin conservation (radiative decay $\Delta^{0} \rightarrow n+\gamma$ takes less than $1 \%$ of total decay width $\Gamma_{\Delta^{0}} \approx 118 \mathrm{MeV}$ ).

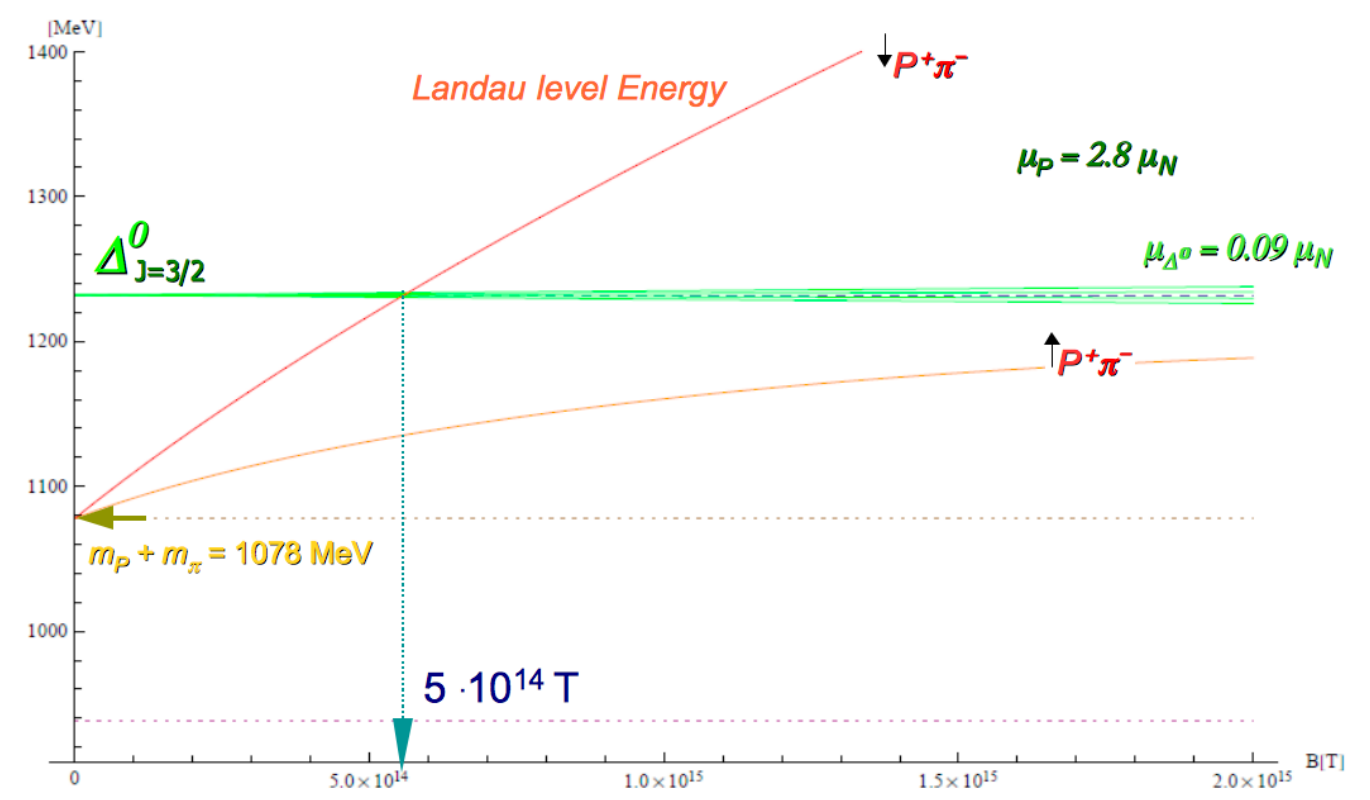

Figure 6: Energy of $\Delta^{0}(1232)$ resonance and of its decay products $P+\pi^{-}$in static magnetic field, using $\mu_{\Delta^{0}}=2 \mu_{d}+\mu_{u}=0.09 \mu_{N}$ and two orientations $s_{z}= \pm 1 / 2$ for the spin of proton with $\mu_{p}=2.79 \mu_{N}$.

In the magnetic field, the energy of decay products $p+\pi^{-}$is rising due to Landau energy shifts of $\pi^{+}$and proton. Only $50 \%$ of $\Delta^{0} \rightarrow p+\pi^{-}$decays become kinematically forbidden in field $B \approx 5 \cdot 10^{14} \mathrm{~T}$ due to spin-dependent interaction of proton magnetic moment $\mu_{p}=2.79 \mu_{N}$ with the external field $B$ (see Fig. 6). Since strong decay channel $\Delta^{0} \rightarrow n+\pi^{0}$ will remain open in the magnetic field, a complete closing of $\Delta^{0} \rightarrow p+\pi^{-}$charged decay channel would not have so interesting consequences, as we have anticipated in the case of $\rho^{0} \rightarrow \pi^{+} \pi^{-}$decay. Radiative and 
Dalitz decays of $\Delta^{0}$ resonance thus cannot be significantly enhanced in the magnetic field by the mechanism we have discussed in the $\rho^{0}$ meson case.

\section{Summary and Conclusions}

We have considered the influence of static magnetic field $B \approx 10^{14}-10^{15} \mathrm{~T}$ on decay of $K^{*}, \Lambda^{*}$, $\Xi^{0 *}, D^{ \pm *}, \Delta^{0}$ and $\rho^{0}(770)$ hadronic states. We conclude, that due to the increase of the lowest $n=0$ Landau energy of charged particles in the magnetic field and due to the interaction of magnetic moments of decaying particles and decay products with the field, specific strong decay channels may become kinematically suppressed or closed. Consequently, isospin conservation in strong decays of $\Delta^{0}, K^{*}, \Lambda^{*}, \Xi^{0 *}$ may become violated, and reconstructed yields of resonances in heavy ion collisions may become systematically biased, if extremal magnetic fields remain present for sufficiently long time.

In the case of $\rho^{0}(770)$ decays we suggest that kinematical suppression of $\rho^{0}(770) \rightarrow \pi^{+} \pi^{-}$ decay channel may lead to substantial enhancement of dilepton decays of $\rho^{0}$ mesons in heavy ion collisions. In Table 1 we show relevant decay channels, critical magnetic field, decay widths and lifetimes of studied resonant states.

\begin{tabular}{ccccc}
\hline \hline & Width $[\mathrm{MeV}]$ & Lifetime $[\mathrm{fm} / \mathrm{c}]$ & $B_{\text {crit }}\left[10^{14} \mathrm{~T}\right]$ & Channel \\
\hline$\rho^{0}$ & 150 & 1.3 & 20 & $\pi^{+} \pi^{-}$ \\
$\Delta^{0}$ & 118 & 1.7 & 5 & $p+\pi^{-}$ \\
$K^{0 *}$ & 50 & 4 & 15 & $K^{-} \pi^{+}$ \\
$\Lambda^{*}$ & 16 & 13 & 5 & $p+K^{-}$ \\
$\Xi^{0 *}$ & 9 & 21 & 4 & $\Xi^{ \pm} \pi^{ \pm}$ \\
$D^{ \pm *}$ & 0.1 & 2040 & 0.3 & $D^{0} \pi^{ \pm}$ \\
\hline
\end{tabular}

Table 1: Parameters and relevant decay channels of selected resonances.

It is important to emphasize, that lifetime of resonances should be comparable to the magnetic field duration for the effects discussed here to be relevant in ultra-relativistic heavy ion collisions. Although total strength of the magnetic field created by the charge of interacting heavy nuclei at LHC and RHIC accelarators is sufficient to induce the effects studied here, the presence of deconfined partonic plasma is necessary to slow down [4] the fast magnetic field decay anticipated in peripheral heavy ion interactions.

\section{Acknowledgments}

This work was supported by Slovak grant agency VEGA under project 2/0197/14. Invitation and hospitality of the organizers of XXII Baldin conference were highly appreciated.

\section{References}

[1] W.-T. Deng and X.-G. Huang, Phys. Rev. C85, p.044907, 2012.

[2] K. Fukushima, D. Kharzeev, H. Warringa, Phys. Rev. Lett. 104, p.212001, 2010.

[3] A.J. Mizher, M.N. Chernodub, E.S. Fraga, Phys. Rev. D82, p.105016, 2010. 
[4] K. Tuchin, Phys. Rev. C82, p.034904, 2010.

[5] M.N. Chernodub, Phys. Rev. D82, p.085011, 2011.

[6] L. D. Landau and E.M. Lifschitz, Course of Theoretical Physics. Vol. 3 (1977).

[7] A.B. Knospe for ALICE collab., Journal of Physics: Conference Series 446, 012056, 2013; B. Abelev et al. (ALICE collaboration), arXiv:1404.0495.

[8] A.M. Torres et al., European Physics Journal A48, 185, 2012.

[9] D.H. Rischke and M. Gyulassy, Nucl. Phys. A597 p.701, 1996; R. Sahoo, T.K. Nayak, J. Alam, B.K. Nandi, S. Kabana, arXiv:1007.4335.

[10] L. Gaudichet for STAR coll., Jour. of Phys. G30 Nucl. Part. Phys. S549, 2004.

[11] B. Abelev et al. (STAR collaboration), Phys. Rev. Lett. 97 p.132301, 2006.

[12] B.O. Kerbikov and L.V. Malinina, Phys. Rev. C81 p.034901, 2010. 